\title{
Leishmania turns down the heat
}

The authors

propose that

CD200 is induced

in response to

L. amazonensis

infection and...

suppresses

production of the

microbicide NO.
Millions of people worldwide are infected by parasites from the genus Leishmania; the severity of symptoms varies greatly, from deadly visceral infections to self-healing skin lesions, depending on the infecting species. However, the mechanisms behind the differences in pathogenicity of Leishmania spp. are unclear. Now, Cortez et al. reveal one mechanism behind these differences, showing that Leishmania amazonensis, which causes severe cutaneous lesions, induces host expression of the macrophage inhibitor CD200, whereas Leishmania major, which causes milder lesions, does not.

The cell surface glycoprotein CD200 engages its receptor, CD200R, to generate an inhibitory signal that damps down local macrophage activation as part of a mechanism to prevent the damage that can be caused by sustained inflammation in response to self and non-self antigens. When performing global gene expression analysis of L. amazonensis-infected bone marrow macrophages (BMMs), the authors noticed a spike in CD200 expression 1 hour after infection. To investigate the role of CD200 in L. amazonensis infection, they infected BMMs from CD200-null mice with L. amazonensis and found that the parasite could not replicate; however, addition of exogenous CD200 to CD200-null BMMs rescued parasite replication. These experiments suggested a crucial role for CD200 in the survival and pathogenesis of L. amazonensis.

Next, the authors tested the role of CD200 during infection in vivo. They found that wild-type mice infected with L. amazonensis developed fastprogressing, non-healing lesions, whereas infected CD200-null mice

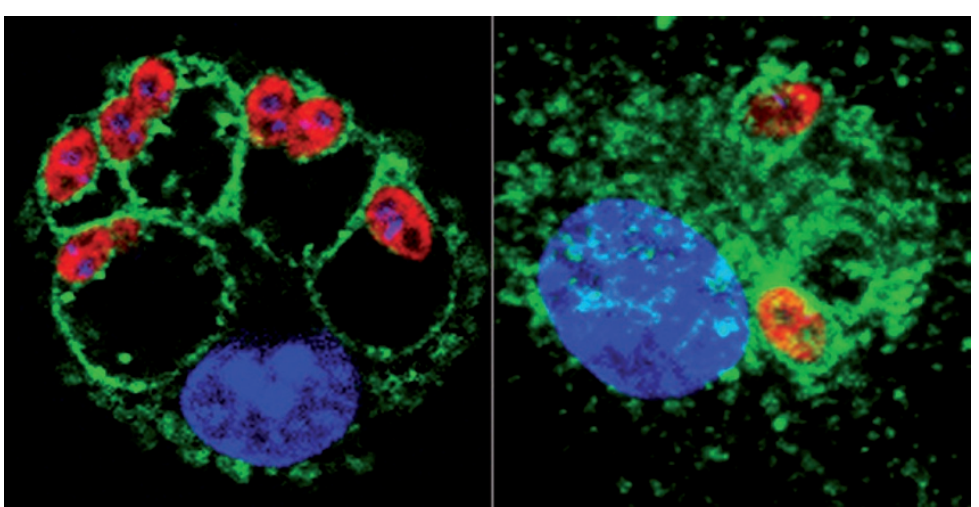

Mouse macrophages that have been infected with Leishmania amazonensis for 48 hours; wild-type macrophages are shown on the left, and CD200-null macrophages on the right. As the parasites cannot replicate in CD200-null macrophages, they appear as individual parasites in small intracellular vacuoles. Red, antibodies specific to Leishmania spp.; green, the lysosomal marker LAMP1; blue, DAPI $\left(4^{\prime}, 6\right.$-diamidino-2-phenylindole) staining of the host cell and parasite DNA. Image courtesy of Mauro Cortez, University of Maryland, USA.

developed smaller lesions that healed over time, probably because (similarly to the BMM results) the parasite could only efficiently replicate in the wild-type mice.

The related parasite L. major causes smaller cutaneous lesions than L. amazonensis, and these lesions tend to be self-healing. Cortez et al. found that L. major infection did not increase CD200 expression, and lesions in wild-type and CD200-null mice infected with L. major healed at the same rate; furthermore, parasite replication was unaffected by the $C d 200$ deletion. However, adding exogenous CD200 protein to both wild-type and CD200-null L. major-infected BMMs significantly increased parasite replication, and this treatment also increased lesion size in L. major-infected mice.

It is not known exactly how L. amazonensis induces CD200 or how CD200 increases leishmanial virulence. However, previous studies have shown that the production of nitric oxide (NO) by inducible NO synthase (iNOS) is a key component of the initial innate immune response to infection with leishmanial parasites. The authors found that iNOS expression levels were higher in CD200-null BMMs than in wild-type BMMs following L. amazonensis infection, and that treatment of CD200-null BMMs with an iNOS inhibitor rescued the ability of parasites to replicate. These results suggest that the inhibition of NO production is an important component of leishmanial virulence. The authors propose that CD200 is induced in response to L. amazonensis infection and interacts with CD200R on nearby macrophages, decreasing their chances of activation and suppressing the production of the microbicide NO.

Joanna E. Huddleston

ORIGINAL RESEARCH PAPER Cortez, M. et al. Leishmania promotes its own virulence by inducing expression of the host immune inhibitory ligand CD200. Cell Host Microbe 9, 463-471 (2011) FURTHER READING Kaye, P. \& Scott P.

Leishmaniasis: complexity at the host-pathogen interface. Nature Rev. Microbiol. 9, 604-615 (2011) 